\title{
LESÕES DENTÁRIAS ORIUNDAS DA OCLUSÃO
}

Laio da Costa DUTRA ${ }^{1}$

Eduardo José Guerra SEABRA²

Alexandre Policarpo da SILVA

Yuri Victor de Medeiros MARTINS ${ }^{4}$

Gláucya Raquel Souza da Fonsêca DUTRA ${ }^{5}$

${ }^{1}$ Cirurgião-dentista. Doutorando em Odontologia do Programa de Pós-Graduação em Odontologia da Universidade Estadual da Paraíba - PPGO/UEPB. Email: laiodutra@gmail.com

${ }^{2}$ Cirurgião-dentista. Doutor em Ciências da Saúde pela Universidade Federal do Rio Grande do Norte - UFRN. Professor Adjunto IV do Curso de Odontologia da Universidade do Estado do Rio Grande do Norte. Email: ejgseabra@yahoo.com.br.

${ }^{3}$ Cirurgião-dentista. Mestre em Saúde e Sociedade pela Universidade do Estado do Rio Grande do Norte PPGSS/UERN. Professor Substituto do Curso de Odontologia da Universidade do Estado do Rio Grande do Norte UERN. Email: policarpogande@hotmail.com

${ }^{4}$ Cirurgião-dentista. Mestre em Saúde e Sociedade pela Universidade do Estado do Rio Grande do Norte PPGSS/UERN. Email: yurivictormm@gmail.com

${ }^{5}$ Enfermeira. Graduada em Enfermagem pela Universidade do Estado do Rio Grande do Norte - UERN. Especializanda em Urgência, Emergência e UTI pela Faculdade Integrada de Patos - FIP. Email: glaucyra@ hotmail.com.

Recebido em: 02/09/2015 - Aprovado em: 03/01/2016 - Disponibilizado em: 30/07/2016

RESUMO: Descrever as lesões dentárias geradas por de agentes físicos, como a abrasão dentária, abfração, bruxismo e apertamento. Realizou-se a pesquisa nas bases de dados eletrônicos Scielo, Pubmed, Lilacs, BBO. Foram encontrados artigos específicos sobre o assunto utilizando os seguintes descritores: abrasão dentária, desgaste dos dentes, bruxismo, colo do dente, oclusão dentária. Apresentando-se com aspecto arredondado ou em forma de valas, a lesão de abrasão é encontrada nas superfícies vestibulares na região da junção cemento esmalte, sendo a cavidade assintomática e com superfície polida, possuindo como causa mais comum a escovação traumática. A lesão de abfração se apresenta em forma de cunha, com bordas vivas e término cavitário nítido, geradas por forças oclusais aplicadas excentricamente, mal dirigidas no elemento dentário,em que a tensão de tração é concentrada no fulcro cervical, ocasionando a rupturada união química dos cristais de hidroxiapatita do esmalte. O bruxismo cêntrico (apertamento) produz facetas com aspecto irregular e superfícies rugosas, geralmente não coincidentes e sem brilho. O bruxismo excêntrico (ranger de dentes) apresenta desgastes com superfície achatada, polida, lisa e localizam-se em pontos bem definidos de dentes antagonistas. Os bruxismos cêntrico e excêntrico possuem etiologia multifatorial. O conhecimento sobre a etiologia, os aspectos clínicos e as consequiências destas lesões quando não tratadas, resultarão em um prognóstico positivo, proporcionando um correto planejamento do tratamento odontológico.

Palavras-chave: Abrasão dentária. Bruxismo. Colo do dente. Desgaste dos dentes.Oclusão Dentária.

\section{DENTAL INJURIES ORIGINATED BY OCCLUSION}

ABSTRACT: Describe the dental injuries generated by physical agents such as dental abrasion, v-shaped lesions, bruxism and clenching. Was conducted research in electronic databases SciELO, PubMed, Lilacs, BBO. They found specific articles on the subject using the following key words: dental abrasion, wear of the teeth, bruxism, tooth neck, 
dental occlusion. Presenting a rounded or shaped ditches, abrasion damage is found on the buccal surfaces on the enamel-cement junction region, being asymptomatic and polished surface cavity, possessing as the most common cause of traumatic brushing. V-shaped lesions presents wedge-shaped, with sharp edges and end sharp cavity generated by occlusal forces applied eccentrically misdirected the tooth, wherein the tensile stress is concentrated at the cervical fulcrum, causing breakage of the chemical bond the enamel hydroxyapatite crystals. The centric bruxism (clenching) produces veneers with irregular appearance and rough surfaces, generally do not coincide and dull. The eccentric bruxism (teeth grinding) shows wear with flat surface, polished, smooth and are located at defined positions antagonists teeth. The centric and eccentric bruxism have multifactorial etiology. The knowledge about the etiology, clinical aspects and consequences of these lesions when untreated, will result in a positive outcome, providing a correct planning of dental treatment.

Keywords: Tooth Abrasion. Bruxism. Tooth Cervix. Tooth Wear. Dental Occlusion

\section{INTRODUÇÃO}

Nos últimos anos vem ocorrendo um aumento considerável na expectativa de vida da população no Brasil, alcançando no ano de 2013, 74,9 anos (IBGE, 2014). Este aumento está associado a um melhor controle preventivo de doenças orais e sistêmicas, provocando um aumento da qualidade de vida da população (TAVARES et al., 2007).

É cada vez mais frequente a permanência dos dentes permanentes em indivíduo com o avançar da idade, portando se mostra de grande importância a manutenção e promoção de saúde desses dentes, como também de sua saúde bucal. Ao longo do tempo, as estruturas dentárias vão sofrendo processos fisiológicos ou patológicos que alteram a sua constituição, e dentre os principais processos que acometem essas estruturas, tem-se o desgaste dentário. Este varia de acordo com a fonte que o provocou, sendo cumulativos e irreversíveis durante a evolução da idade (SANTOS et al., 2009), podendo provocar alteração no esmalte, na dentina e na polpa dentária.
O Sistema Estomatognático

(SE)

desempenha atividades funcionais ou fisiológicas, sendo os atos de mastigar, falar e deglutir, os quais são determinados por reflexos protetores e músculos. Estas ações fazem com que ocorra um desempenho normal das funções do SE, evitando patologias, como os hábitos parafuncionais (atividades não interrompidas pelos contatos dentais), que podem gerar movimentos não fisiológicos causando contração muscular desnecessária, podendo desenvolver o quadro de bruxismo (apertar e/ou ranger os dentes) (PRIMO; MIURA e BOLETA-CERANTO, 2009).

As facetas de desgaste dentárias são achados comuns, em que podem se apresentar em pequenas proporções atingindo o esmalte ou provocando grandes destruições acometendo a dentina e esmalte, variando entre desgastes localizados (em um elemento dentário) e generalizados, possuindo uma etiologia associada a vários fatores que podem se apresentar em conjunto ou não, como: hábitos parafuncionais, regurgitação ácida, fatores diários da dieta, interferências e 
traumas oclusais (LOPES et al., 2007).

Objetivou-se, através de uma revisão de literatura, descrever os desgastes dentários provocados por agentes físicos, tais como: abrasão dentária, abfração, facetas de desgaste por bruxismo e apertamento.

\section{METODOLOGIA}

Para o desenvolvimento deste estudo, se realizou pesquisas nas principais bases de dados eletrônicos Scielo, Pubmed, Lilacs, BBO. Os descritores utilizados foramabrasão dentária, desgaste dos dentes, bruxismo, colo do dente e oclusão dentária. A partir da lista de artigos, foram aplicados critérios de inclusão para a escolha dos artigos como: Estudos que abordam conceitos, etiologia e características clínicas dos desgastes dentários provenientes da oclusão. Os artigos que não possuíam estes critérios foram excluídos da pesquisa. Foram selecionados artigos em língua portuguesa, espanhola e inglesa.

\section{LESÃO DE ABRASÃO}

A abrasão é uma lesão oriunda do atrito entre o dente e o um agente exógeno (DZAKOVICH e OSLAK, 2008), gerando um processo de desmineralização ou perda patológica da estrutura dentária ou restauração, livre de biofilme dental, que ocorre de maneira lenta, gradual e progressiva (AMARAL et al., 2012), sendo produzida por uma atividade funcional anormal, estando associada ao mecanismo de estresse mecânico (MOLENA et al., 2008).

O padrão da lesão é normalmente determinado pelo objeto agressor (MEHTA et al., 2012). A causa mais comum deabrasão é a escovação traumática, que combina um agente abrasivo dentário com a uma pressão excessiva durante a escovação dos dentes (LATORRE et al., 2010), como também outros processos mecânicos anormais, que envolvem objetos estranhos ou substâncias constantemente introduzidas na boca em contato com o dente (MOLENA et al., 2008; LIU et al., 2014), como o uso indevido defio dental,palitos de dente (PEREZ et al., 2012) e hábitos bucais danosos como mordidade tachas, pregos, alfinetes, linhas, uma hastede tubo, grampos de cabeloouum instrumento de sopro (MEHTA et al., 2012). Os alimentos grosseiros quando são forçados contra a superfície do dente, podem causar lesão de abrasão, podendo ocorre perda de cemento e de dentina interproximal. (LATORREet al., 2010).

Os principais fatores relacionados aos procedimentos de higiene bucal envolvem técnica, frequência, tempo e força aplicada durante o processo de escovação (MOLENA et al., 2008), como também a dureza dos filamentos da escova e forma das terminações dos filamentos (AMARAL et al., 2012).

A abrasão produzida pela escovação traumática aparece em forma de ranhuras 
horizontaisna superfície vestibular, podendo ocorrer o aparecimento de cemento e/oudentina (LATORREet al., 2010), quando o desgaste se desenvolveu de forma intensa, provocando ranhuras com profundidade considerável (Figura 1). As lesões se apresentam em aspecto arredondado ou em forma de valas encontradas nas superfícies vestibulares na região da junção cemento esmalte (MEHTA et al., 2012), proporcionando um aspecto de pires (Figura 2), sendo cavidades indolores com superfícies polidas (PEREZ et al., 2012).

FIGURA 1: Lesão de abrasão com aspecto de ranhuras horizontais na superfície vestibular de incisivos centrais superiores.

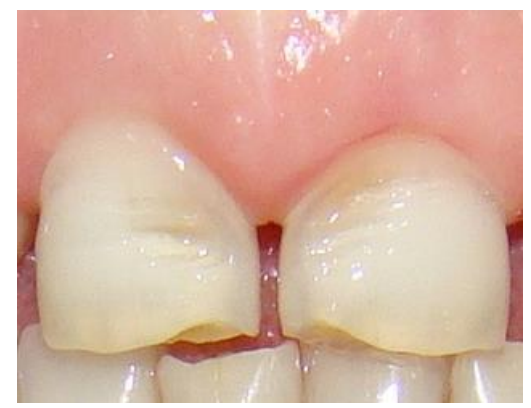

Fonte: Arquivo do autor

O caninoe pré-molar são os dentes mais afetados (MEHTA et al., 2012), sendo o lado oposto ao da mão dominante o que ocorre o maior dano (LATORREet al., 2010).
FIGURA 2: Lesão de abrasão em forma de vala no terço cervical da superfície vestibular de pré-molar superior.

Fonte: Arquivo do autor

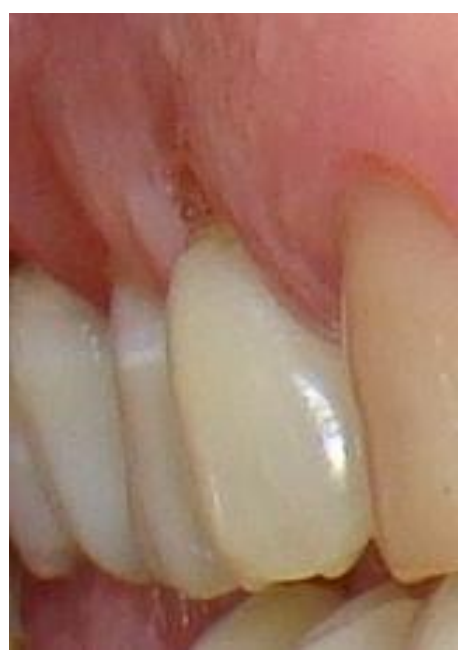

\section{LESÃO DE ABFRAÇÃO}

A abfração é uma lesão que gera perda de estrutura dentária (OLIVEIRA, DAMASCENA, SOUZA, 2010;LATORREet al., 2010; GRIPPO, SIMRING e COLEMAN, 2012;AMARAL et al., 2012) à distancia dos pontos de contatos oclusais (OLIVEIRA, DAMASCENA e SOUZA, 2010).

Esta perda ocorre quando forças oclusais são aplicadas excentricamente, mal dirigidas, no elemento dentário, em que a tensão de tração é concentrada no fulcro cervical, levando a rupturada união química dos cristais de hidroxiapatita do esmalte (LIMA, HUMEREZ FILHO e LOPES, 2005; MOLENA et al., 2008; PEREIRA et al., 2008; LATORREet al., 2010;OLIVEIRA, DAMASCENA e SOUZA, 2010;AMARAL et al., 2012; PEREZ et al., 2012) e consequentemente perda de substância nas 
áreas cervicais dos dentes (AMARAL et al., 2012), principalmente no limite amelocementário (MOLENA et al., 2008).

A perda de substância também pode ser consequência de uma sobrecarga oclusal (MOLENA et al., 2008).Estas lesões cervicais são comumente encontradas nas superfícies vestibulares dos dentes, sendo de difícil ocorrência nas superfícies ligual e proximal (PEREIRA et al., 2008), possuindo maior prevalência na superfície vestibular (Figura 3 e Figura 4). Os dentes com mobilidade apresentam um menor acometimento pelas lesões de abfração do que os sem mobilidade(PEREIRA et al., 2008).

A abfração pode afetar apenas um dente e clinicamente apresentam-se em forma de cunha ou em forma de $\mathrm{V}$, com arestas vivas e término cavitário nítido (MOLENA et al., 2008; AMARAL et al., 2012; MEHTA et al., 2012; TRENTIN, BERVIAN, 2014). Estes aspectos foram observados nas lesões, possuindo término cervical nítido com a lesão em forma de cunha clássica (Figura 3 e Figura 4). Possui maior prevalência nos dentes inferiores, pois possuem menor diâmetro coronário na região cervical (AMARAL et al., 2012).
FIGURA 3: Lesão de Abfração acometendo pré-molar inferior, possuindo aspecto de cunha com término cervical nítido.

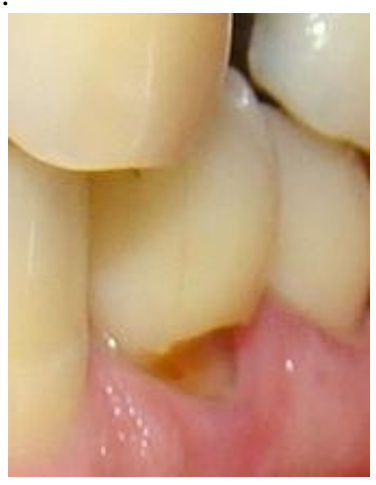

Fonte: Arquivo do autor

A extensãodas lesões édependente do tamanho, duração, direção, frequênciae localizaçãodas forças oclusais (MEHTA et al., 2012).

FIGURA 4: Lesão de Abfração acometendo Canino superior, possuindo aspecto de cunha com término cervical nítido.

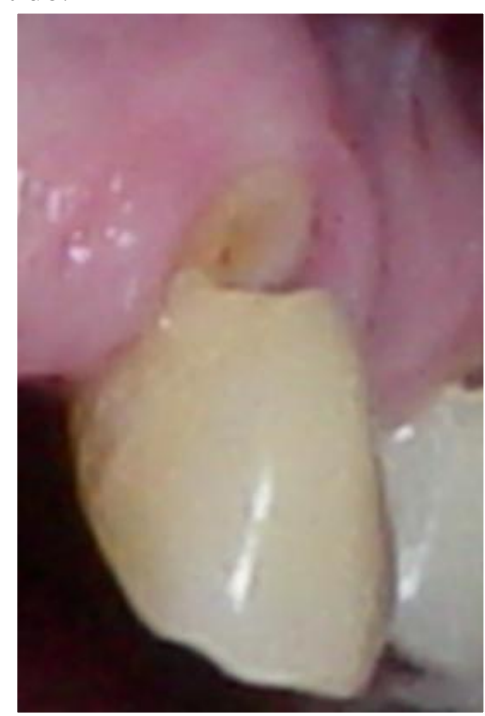

Fonte: Arquivo do autor

\section{BRUXISMO EXCÊNTRICO

O bruxismo é uma atividade parafuncional caracterizada pelo contato não funcional dos dentes, que pode ocorrer de 
forma consciente ou inconsciente (MACHADO et al., 2011).

Este contato não funcional dos dentes se apresenta normalmente como apertamento dentário (bruxismo cêntrico), ocorrendo com maior prevalência durante o dia (bruxismo diurno). O excêntrico, outra forma de apresentação do bruxismo, desenvolve-se através do ranger de dentes, promovendo ruídos (ALMEIDA et al., 2010), sendo mais prevalente durante a noite (bruxismo do sono) (SERAIDARIAN, ASSUNÇÃO e JACOB, 2001; SHETTY et al., 2010; FERINISTRAMBI et al., 2011).

O bruxismo cêntrico e o excêntrico podem ocorrer em horários inversos ou mesmo em associação durante a atividade parafuncional.

(SERAIDARIAN, ASSUNÇÃO e JACOB, 2001). Não é caracterizada como doença, mas quando o quadro clínico exacerba, pode ocorrer um desequilíbrio fisiopatológico do SE (MACHADO et al., 2011).

O bruxismo possui uma etiologia multifatorial, sendo uma desordem complexa e com frequência, difícil de ser identificada (RODRIGUES et al., 2006). PRIMO, MIURA, e BOLETA-CERANTO (2009) relatam como favorecedores do desenvolvimento do bruxismo as características morfológicas, fatores psicossociais e genéticos.

FERNANES NETO (2006) cita que fatores intra-bucais (contato prematuro e interferência oclusal) e extra-bucais (psicológicos, sistêmicos e uso de drogas) poderão ser responsáveis pelo início do bruxismo. Mesmo com todos estes fatores citados na literatura, a etiologia do bruxismo ainda é controversa (KATAOKA et al., 2015).

O bruxismo pode provocar sintomatologia muscular (fadiga, aumento do grau de tensão dos músculos mastigatórios, mialgia, miosite, espasmo, contratura, dores na musculatura cervical e postural), trauma em estruturas anatômicas da ATM (fibras elásticas posteriores da cápsula, disco articular, desvios laterais, desgastes articulares) e estruturas auriculares (causando uma falsa sensação de obstrução)(SOARESet al., 2004; PRIMO, MIURA e BOLETACERANTO, 2009; BLINI et al., 2009; ALMEIDA et al., 2010).

Além destas alterações, o bruxismo pode causar desgaste dental, que é uma modificação cumulativa de atividades funcional e parafuncionais, e os fatores como idade, gênero, oclusão, dieta e ingestão de bebidas ácidas podem estar associados (SHETTY et al., 2010; DE LA HOZAIZPURUA et al., 2011).

Os desgastes podem apresentar diferentes graus de intensidade, desde esmalte até grandes exposições de dentina, podendo ocorrer fraturas e até perda de restaurações(PRIMO, MIURA e BOLETACERANTO, 2009), corroborando com os 
graus de destruição dentária encontrados, em que são visíveis as grandes exposições de dentina (Figura 5) como também destruição e perda de restauração (Figura 6).

FIGURA 5: Paciente com facetas de desgaste por atividade de bruxismo cêntrico e bruxismo excêntrico.

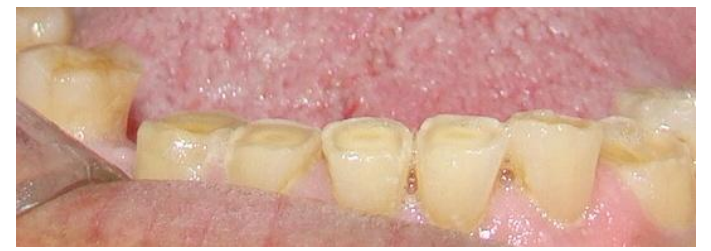

Fonte: Arquivo do autor

As facetas de desgaste geradas pelo bruxismo excêntrico (Figuras 5 e 6 ) se apresentam como superfície achatada, polida, lisa e localizam-se em pontos bem definidos de dentes antagonistas, e as de bruxismo cêntrico (Figura 5), são irregulares, rugosas, geralmente não coincidentes e sem brilho (ANTONIO, PIERRO e MAIA, 2006; PAIVA et al., 2008; DINIZ, SILVA e ZUANON, 2009).

FIGURA 6: Paciente com facetas de desgaste por atividade de bruxismo excêntrico.

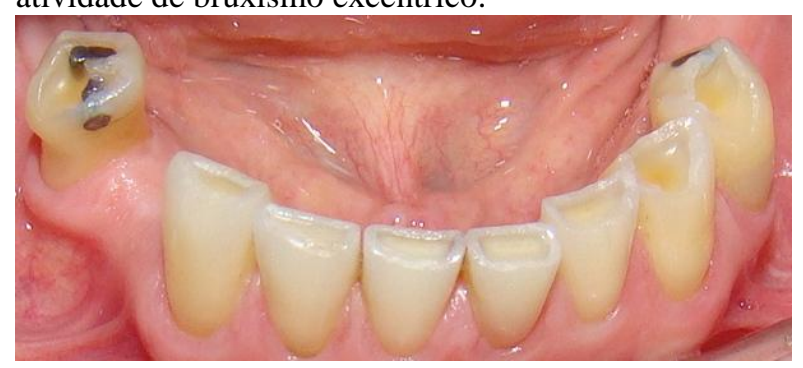

Fonte: Arquivo do autor

\section{CONCLUSÃO}

As forças oclusais excêntricas, escovação traumática e outros processos mecânicos podem causar perda de estrutura do dente na região cervical. Com o conhecimento dos aspectos clínicos e etiologia das lesões de abrasão e abfração, pode-se realizar um correto diagnóstico, propiciando a interrupção do curso destas alterações.

Devido à etiologia multifatorial e várias características clínicas, a realização do diagnóstico correto de bruxismo não é um procedimento fácil, pois necessita de um exame clínico e físico detalhados, buscando de forma minuciosa informações que possam levar ao diagnóstico preciso.

Então, o conhecimento do cirurgião dentista sobre a etiologia, os aspectos clínicos e as consequências de quando não se programa o tratamento, resultarão em um prognóstico positivo, proporcionando um correto planejamento do tratamento odontológico, sendo assim, servirá como fomento para uma melhor conduta clínica diante destes casos.

\section{REFERÊNCIAS}

ALMEIDA, F. L. et al. Relation between pain and electric activity in the presence of bruxism. Rev. CEFAC, v. 13, n. 3, p. 399406, 2011.

AMARAL, S. M. et al. Lesões não cariosas: o desafio do diagnóstico multidisciplinar. Arq. Int. Otorrinolaringol., São Paulo - Brasil, v.16, n.1, p. 96-102, Jan/Fev/Mar, 2012.

ANTONIO, A. G.; PIERRO, V. S. S.; MAIA, L. C. Bruxism in Children: A Warning Sign 
for Psychological Problems. J Can Dent

Assoc. v.72, n.2, p.155-160, 2006.

BARATIERI, L. N. Dentística: fundamentos

e possibilidades. São Paulo: Ed. Santos;

2001, 739p.

BLINI, C. C. et al. The relationship between the bruxism and the severity of symptoms in the temporomandibular disorder. Revista CEFAC, v. 12, n. 3, p. 427-433, mai/jun, 2010.

BRASIL. Instituto Brasileiro de Geografia e Estatística. Tábuas Completas de Mortalidade do Brasil - 2013. Rio de Janeiro: IBGE; 2014. Disponível em: http://teen.ibge.gov.br/noticias-teen.html, acesso em 28/08/2015.

DE LA HOZ-AIZPURUA, J. L. et al. Sleepbruxism. Conceptual review and update.Med Oral Patol Oral Cir Bucal,v. 16, n. 2, p. 231-8, mar., 2011.

DINIZ, M. B.; SILVA, R. C.; ZUANON, A. C. C.Bruxismo na infância: um sinal de alerta para odontopediatras e pediatras. Rev Paul Pediatr, v. 27, n. 3, p. 329-3

34, 2009.

DZAKOVICH, J. J.; OSLAK, R. R.In vitro reproduction of noncarious cervical lesions.Journal of Prosthetic Dentistry, v. 100, n. 1, p. 1-10, 2008.

FERINI-STRAMBI, L. et al. Bruxism and nocturnal groaning.ArchivesItaliennes de Biologie, v. 149, n. 4, p. 467-477, 2011.

FERNANES NETO, A. J. Roteiro Oclusão.

DisfunçãoDentária. Capítulo 8, 2006.

GRIPPO, J. O.; SIMRING, M.; COLEMAN, T. A. Abfraction, Abrasion, Biocorrosion, and the Enigma of Noncarious Cervical Lesions: A 20-Year Perspective. Journalof Esthetic and Restorative Dentistry, v. 24, n. 1, p. 1023, 2012.
KATAOKA, K. et. al. Association Between Self-Reported Bruxism and Malocclusion in University Students: A Cross-Sectional Study. Journal of Epidemiology, v. 25, n. 6, p. 423-430, 2015.

LATORRE, C. et al. Desgaste dental y factores de riesgoasociados. Rev.CESOdont, v. 23, n. 1, p.29-36, 2010.

LIMA, L. M.; HUMEREZ FILHO, H.; LOPES, M. G. K. Contribution to the study of the prevalence, distinguished diagnosis and etiologic factors of the not carious cervical injuries.RSBO v. 2, n. 2, p. 17- 21, 2005.

LIU, B. et al. Tooth wear in aging people: an investigation of the prevalence and the influential factors of incisal/occlusal tooth wear in northwest China. BMC oral health, v. 14, n. 1, p. 1-5, 2014.

LOPES, F. A. M. et al. Prevalence of dental wear and their relation to occlusal aspects and parafunctional habits. Rev Odontol UNESP, v. 36, n. 1, p. 47-52, 2007.

MACHADO, E. et al. Bruxismo do sono: possibilidades terapêuticas baseadas em evidências. Dental Press J Orthod, v. 16, n. 2, p. 58-64, mar./apr.,2011.

MEHTA, S. B. et al. Current concepts on the management of tooth wear: part 1.

Assessment, treatment planning and strategies for the prevention and the passive management of tooth wear.British Dental Journal, v. 212, n. 1, p. 17-27, jan., 2012.

MOLENA, C. C. L. et al. Non-carious lesions in elderly. Rev. Bras. Cir. CabeçaPescoço, v. 37, n. 3, p. 152-155, 2008.

MOLENA, C. C. L. et al. Relationship between non-carious cervical lesions and habits. Rev. Bras. Cir. Cabeça Pescoço, v. 
37, n. 4, p. 206-211, 2008.

PAIVA, H. J. et al. Noções e Conceitos

Básicos em Oclusão, Disfunção

Temporomandibular e Dor Orofacial.

Editora Santos, 2008, 458p.

PEREIRA, A. F. V. et al. Abfraction lesions reviewed: current concepts. RGO, v. 56, n.3, p. 321-6,2008.

PEREZ, C. R. et al. Restoration of

Noncarious Cervical Lesions:When, Why, and How. International Journal of Dentistry. v. 2012, p. 1-8, 2012.

PRIMO, P. P.; MIURA, C. S. N.; BOLETACERANTO, D. C. F.

Hysiopathologicconsiderationsaboutbruxism. Arq. Ciênc. Saúde UNIPAR, v. 13, n. 3, p. 263-6,2009.

OLIVEIRA, A. C. S.; DAMASCENA, N. P.; SOUZA, C. S. Clinical analysis of patients with non-carious cervical lesions and its relation to habits. Rev Sul-Bras Odontol. v. 7, n. 2, p. 182-192, jun., 2010.

RODRIGUES, C. K. et al. Bruxismo: uma revisão da literatura. Publ. UEPG Ci. Biol. Saúde, v. 12, n. 3, p. 13-21, set. 2006.

SANTOS, T. S. et al. Relationship of Tooth Wear to Temporomandibular Dysfunction.

Rev. Cir. Traumatol. Buco-Maxilo-facial, v.9, n.2, p. 73-80, abr./jun., 2009.

SERAIDARIAN, P. I.; ASSUNÇÃO, Z. L. V.; JACOB, M. F. Bruxism: An Update of Concepts, Etiology, Prevalence and Management. Jornal Brasileiro de Oclusão, ATM e Dor Orofacial, v.1, n.4, p. 290-5, out./dez., 2001.

SHETTY, S. et al. Bruxism: A Literature Review. J Indian Prosthodont Soc., v. 10, n. 3, p. 141-8, jul./set., 2010.

SOARES, I. S. Q. et al. Bruxismo: desempenho da mastigação em adultos jovens. Rev CEFAC, v.6, n.4, p. 358-362, out./dez., 2004.

TAVARES, M. J. G. M. et al. Characterization of tooth wear in patients above 35 years of age. RGO, v. 55, n 1, p. 4753, jan./mar. 2007.

TRENTIN, M. S.; BERVIAN, J. Hipersensibilidade dentinária cervical: uma revisão da literatura. RFO, v. 19, n. 2, p. 252257, mai./ago., 2014. 